\title{
Some large deviation estimates for an Erlang queue of phosphorylated T-cell receptors
}

\author{
Janak R. Wedagedera* \\ Department of Mathematics, Faculty of Science, University of Ruhuna, Matara.
}

Revised: 25 May 2010 ; Accepted: 17 September 2010

\begin{abstract}
T-cell activation can be viewed as a stochastic process occurring over a finite time interaction with an antigen presenting cell (APC). T-cell commits to activation based on a finite number of T-cell receptor (TCR) phosphorylation events; the high specificity and sensitivity of T-cells indicating that information on ligand quality can be extracted with precision. In this paper, a certain (Erlang) queue model is proposed for T-cell activation, which is treated under two criteria or strategies that utilize the history of TCR phosphorylation to discriminate ligands. The major result obtained in this paper in the above context is the determination of the most probable way the phosphorylated TCRs achieve a given threshold (for signal strength) under two particular strategies, using WentzellFreidlin theory of large deviations for processes.
\end{abstract}

Keywords: T-cell activation, TCR-pMHC interaction, variational problem for most probable path, Wentzell-Freidlin theory.

\section{INTRODUCTION}

T-cells [ Cytotoxic T-Lymphocytes (CTL) ] play a major role in the adaptive immune system of vertebrates in defense against viral, bacterial and fungal infections. CTLs reside in tissues or circulate through the body via the blood and lymph to detect cells that have been compromised by foreign organisms (Chao et al., 2003; Lanzavecchia, 1997; McKeithan, 1995).

In order to launch an immune attack on invading pathogens, the T-cell should be able to recognize the pathogen to which the T-cell is specialized. This specialization or specificity is based on the diversity of T-cells when they developed in the bone-marrow. In addition, the T-cell should also be incapable of acting against self peptides. The T-cells that do not have this capability are eliminated in the thymus, which is known as thymic selection (Roitt, 1998).
A T-cell, which has passed through the thymic selection test but had not encountered a derived peptide of an antigen [or antigen peptide - the smallest part prepared by an antigen presenting cell (APC)] of an invading pathogen is called a naive T-cell. (There is a spectrum of peptides presented on the surface of APCs, including the peptides derived from self proteins).

The interaction between the T-cell and the APC is mediated by T-cell receptor molecules (TCR) and APC's specialized antigen-presenting receptor encoded by the major histocompatibility complex (MHC) of genes. This complex contains the $\mathrm{pMHC}$ molecule, the peptide which is trapped in the MHC molecule. A T-cell is said to patrol through the blood and lymphoid organs of the body in search of specific peptides. When an APC encounters the T-cell, the TCRs in the contact area between the two cells bind to the pMHC complexes. This initiates the process whereby the transmembrane molecules associated with the TCR acquire the ability to transmit signals to the cellular interior by phosphorylation of intracellular signaling domains associated with the TCR/CD3 complex (Palmer \& Werlen, 2002) (Figure 1 - Left).The triggering of TCRs leads to various cellular responses, such as changes in gene expression or the killing of a target cell (Parham, 2000), after initiating sustained signalling to the nucleus.

As seen in Figure 1 - Right, the diversity of the peptide-antigens is illustrated by various geometrical shapes of the molecules. In this model (based on the serial triggering hypothesis (Valitutti et al., 1995 :Valitutti \& Lanzavecchia, 1997) a single TCR may encounter more than one $\mathrm{pMHC}$ between the contact areas. This variation in computations is quantified by the dissociation rate of a peptide when it is bound to a TCR, as described in 

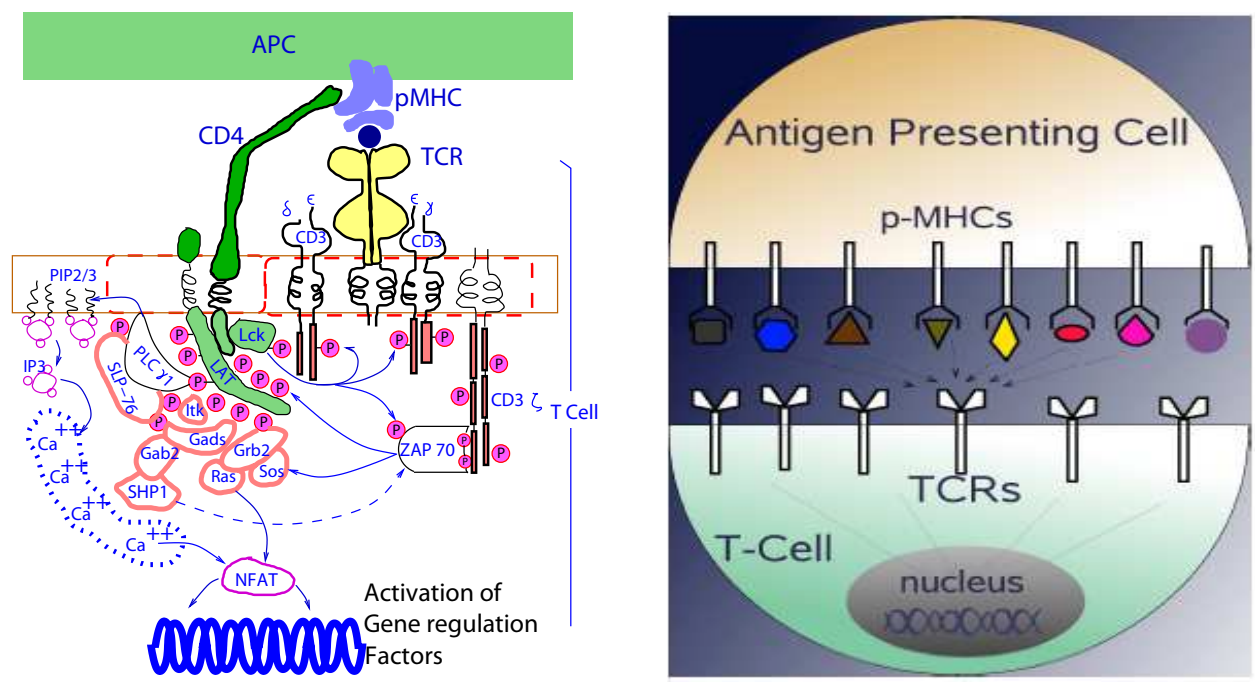

Figure 1: Left: The T-cell receptor (TCR) interacts with an antigenic peptide-MHC complex, and the co-receptor (CD4 or CD8) stabilizes this interaction by bringing the kinase LCK in the proximity of the TCR-CD3 complex. LCK can then phosphorylate the CD3-associated immunoreceptor tyrosine-based activation motifs (ITAMs). There are a number of different signalling cascades initiated by these interactions leading to various stages of activation of the T-cell by activating gene regulation factors such as NFAT and NF.

Right: Cartoon of the T cell APC interaction model considered in the paper. The diversity of peptide-antigens is illustrated here by various geometrical shapes of the molecules which is represented by the dissociation rate $k_{\text {off }^{\prime}}$ Source: Valitutti et al., 1995 :Valitutti \& Lanzavecchia, 1997

the text. A TCR thus acts as a signalling device, which sends certain signals to the nucleus via many different pathways in several hierarchies. In the model described in Figure 3.3, $x(t)$ denotes the number of signalling (or triggering) TCRs at time $t$.

The background of T-cell activation, paying attention to threshold and signal duration aspects, is reviewed in order to justify our modelling approach proposed in the paper. Activation of a T-cell by exposure to its specific agonist may ultimately lead to cell proliferation, differentiation and launching cytotoxic attacks on target cells. However, recent experimental data suggests that this is not achieved in a single step, and is a multistep sequence of events where disruption of signalling proportionally reduces activation (Faroudi et al., 2003; Huppa et al., 2003; Jordan et al., 2009).

The process of T-cell activation can be separated into a hierarchy of signalling and cell response. Initially, at the T-cell surface, the TCR interacts with its ligand, (peptide-MHC or pMHC) within the contact interface formed with an APC. Secondly, TCR binding to its specific ligand leads to phosphorylation of the receptor and recruitment of adapters and kinases that comprise the signalling cascade. The third level of signalling is the integration of these signals to ultimately determine cell function; this includes regulation of the cytoskeleton, control of adhesion within the cell: cell contact (Dustin et al., 2004), directed secretion at the interface (Bossi et al., 2002) and gene transcription.

The threshold concept has remained the predominant means of analysis of this activation/signalling sequence, i.e. a T-cell becomes activated (as measured by some function $\mathrm{X}$ ) if the stimulus is above a threshold. In general, a hierarchy of thresholds is observed for different cell responses (Hemmer et al., 1998; Itoh \& Germain, 1997; Valitutti et al., 1996) with good consistency between cells for the relative threshold order: $T_{\text {cytotoxicity }}$ $\ll T_{\text {cytokine production }}<T_{\text {cell proliferation }}$ (where $T$ stands for threshold).

Thresholds, however, fail to account for the growing number of experimental studies that demonstrate a dependence on the temporal sequence of events. The importance of signal duration was recently emphasized by transient APC exposure studies (Gett et al., 2003; van Stipdonk et al., 2003) where T-cell survival correlated with exposure duration. The percentage of activated T-cells was also significantly affected if duration was shorter than 4 hours (Kaech \& Ahmed, 2001; van Stipdonk 
et al., 2001), whilst there is a correlation between degree of proliferation and duration of antigen exposure (Schrum $\&$ Turka, 2002). In addition, T-cells can integrate signals other than a sequence of APC encounters (Gunzer et al., 2000), a phenomenon recently quantified by interrupting the T-cell: APC contact demonstrating that the overall duration of contact is a determinant of activation (Davis, 2009; Faroudi et al., 2003). This supports the earlier hypothesis that a T-cell effectively 'counts' the number of productive TCR-pMHC interactions, a conclusion originally based on the high correlation of cell response to the fraction of down-regulated TCRs (Valitutti et al., 1995).

The balance between the competing demands of specificity and sensitivity has been a fundamental question in T-cell biology for over a decade, the T-cell being sensitive to a single agonist pMHC whilst discriminating between single amino acid peptide variants with dissociation (off) rates varying by $30 \%$ (Kersh et al., 1998).

Stochastic approaches to modelling the T-cell APC interaction have been considered in the past (Chan et al., 2001: Noest, 2000; van den Berg et al., 2001). Despite these models, theoretically, it remains unclear how complex TCR triggering dynamics must be to filter out self peptide noise whilst achieving high sensitivity.

The two main hypotheses considered in this study for T-cell activation are as follows:

1) The triggering TCRs send signals to the cell nuclei (via a number of signalling pathways). The T-cell undergoes a number of stages of activation depending on the strength of the integrated signal received by the nuclei. For the activation event E1 to take place the integrated signal must exceed a certain threshold, $\alpha_{1}$, within some duration, $T_{1}$. This is a strategy used by the T-cell and is supported by the threshold experimental evidences mentioned previously.

2) The T-cell attains a certain threshold within a duration $T$ and maintains that for another duration, $\Delta T$. This is supported by the threshold hypothesis and the experimental evidences mentioned previously.

Thus, in this work the author proposes:

1. A stochastic model to describe the integration of signalling TCRs (which can be used to model both strategies explained above) and,
2. To determine the optimal strategy by estimating the efficiency of each via computation of the probability of activation (as defined in the next section) against the triggering (signalling) rate.

\section{METHODS AND MATERIALS}

Modelling threshold and signal integration: Simple activation models motivated by the dependence of activation on the temporal aspects of the signal are used. The aim is to provide a framework for the analysis of TCR triggering. The present approach is to construct queuing theory models of TCR triggering, incorporating the input of phosphorylated TCRs (from a kinetic proofreading scheme), Poisson input rate, a function of the dissociation (unbinding) rate of TCR denoted by $k_{\text {off }}$, and output via TCR down-regulation.

Since full phosphorylation of a TCR occurs in exponential time, this may be considered as a Poisson event. Thus, the present scenario is equivalent with these assumptions to a queue with Poisson input $\lambda=k_{\text {off }} \mid C^{*}$ where, $\left|C^{*}\right|=$ number of fully phosphorylated pMHC-TCR complexes and Poisson output with rate $\mu x(t)$ :

$\stackrel{\lambda=k_{\mathrm{off}}\left|C^{*}\right|}{\longrightarrow} x(t) \stackrel{\mu x(t)}{\longrightarrow}($ down-regulation $)$

This directly accounts for the noise associated with signalling based on a finite number of signalling molecules. In this study an Erlang queue is used to analyze various activation strategies. It is anticipated that they will be appropriate for analysis of feedback models.

The model is analyzed using a large deviation methodology (path analysis by the Wentzell-Freidlin theory), which allows the most probable path to be determined. This aids understanding and analysis of the behaviour of the activation probabilities with respect to variation of parameters, for instance providing analytically tractable approximations. In particular, this analysis turns out to be useful in understanding how the two above-mentioned strategies perform in recognizing a pathogenic derived antigen-peptide by the T-cell.

A basic queuing model: the criteria for activation: A peptide is characterized by its 'off-rate' (or rate of dissociation upon binding with the T-cell receptor) denoted by $k_{-}$. The following two different activation criteria (or strategies $S_{1}$ and $S_{2}$ ) are defined in order to compare the discriminating ability of a T-cell on different kinds of peptides: 
- $\quad S_{1}: T C R^{*}$ (phosphorylated TCR) $>\alpha$ (threshold) within a time $T$ (scanning time).

- $S_{2}: T C R^{*}>\alpha$ for a period $\Delta T$ starting within $T$ of cell-cell conjugation (min. scanning time).

The situation $S_{1}$ may be considered as a pre-synapse formation event (or an initial threshold, which determines the upregulation of the adhesion molecule LFA1 affinity). $S_{2}$ can be considered as a response from a mature synapse (or particularly a phase in which a sustained calcium signal is required for pMHC and TCR to stay conjugated).

It is necessary to determine the above cellular level thresholds in a situation where the probability of activation with respect to these thresholds are assumed to be low (thus, activation becomes a rare event). In a later section the kinetic proof reading model is considered in order to estimate the input parameters required in the triggering phase.

The problem (in particular the triggering events) is formulated as a queue and the activation probabilities in $S_{1}$ and $S_{2}$ estimated respectively with respect to the number of agonist peptides, values for dissociation rate $k_{\text {of }}$ etc.

Initiating TCR signaling: phosphorylation of the key kinases in kinetic proofreading model: Following the kinetic proofreading scheme (McKeithan, 1995; Hopfield, 1974), an $m$-step phosphorylation process is considered:

Figure 3 illustrates

- (A) Unoccupied TCRs
- $\left(B_{1}\right)$ TCR-pMHC complexes formed with association rate $k_{\text {on }}$ and dissociation rate $k_{\text {off }}$

- $\left(B_{m}\right)$ TCR-pMHC complexes undergoing an $m$-step phosphorylation process with forward rate $k^{*}$, yielding a phosphorylated complex $\mathrm{C}^{*}$

- (C) TCR-pMHC complexes dissociate with rate $k_{\text {off }}$ and TCRs down-regulate with rate $\mu x(t)$ where $x(t)$ is the number of triggering TCRs at time $t$.

The steady state computations give

$$
\left[C^{m}\right]=\left[C^{0}\right]\left(\frac{1}{1+\left(k_{\mathrm{off}} / k\right)}\right)^{m}
$$

where $\left[C^{0}\right]$ and $\left[C^{m}\right]$ denote the initial concentration and the concentration of the fully phosphorylated pMHCTCR complex, respectively.

The dissociation rate $\lambda$ with respect to the number of TCRs at stage $(C)$ is found via $\lambda=k_{\text {off }}\left[C^{m}\right]$. It is assumed that $k_{\text {off }} / k^{*}=1 / m$. As $m \rightarrow \infty,\left[C^{m}\right] \sim\left[C^{0}\right] \mathrm{e}^{-1}$ is obtained.

Let $C^{*}(t)$ be the number of TCR-pMHC complexes involved in signalling after the full phosphorylation at time $t$, and $R$ be the total number of phosphorylated TCRs. The probabilities such as $\mathbb{P}\left[x(t)+C^{*}(t) \geq \alpha R\right.$. where $0<\alpha<1$ are estimated.

After achieving the full phosphorylation $\left(C^{*}\right)$, pMHC breaks-off from the TCR at rate $k_{\text {off }}$. However, the remaining complexes may also contribute to the signalling, as well as the TCR*s themselves, implying that for the activation of the T-Cell, at this stage, both of

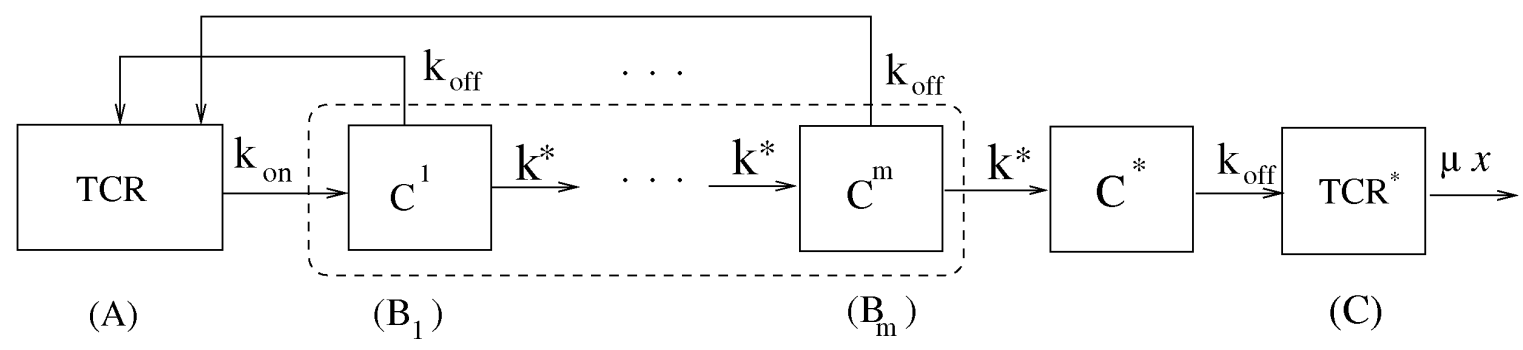

Figure 3: Schematic of an $m$-step phosphorylation reaction process: After the $m$-step phosphorylation process the fully phosphorylated pMHC - TCR complexes dissociate with rate $k_{\text {off }}$, and triggering TCRs [denoted as $T C R^{*}$ ] downregulate with rate $\mu x(t), x(t)$ is the number of triggering TCRs at time $t$. 
these contributions have to be considered. However, the realistic assumption that $k^{*} \ll k_{\text {off }}$ or $C^{*}(t) \ll x(t)$, is made, which will simplify the task of estimating the above probabilities, requiring the estimation of $\mathbb{P}[x(t) \geq \alpha R]$.
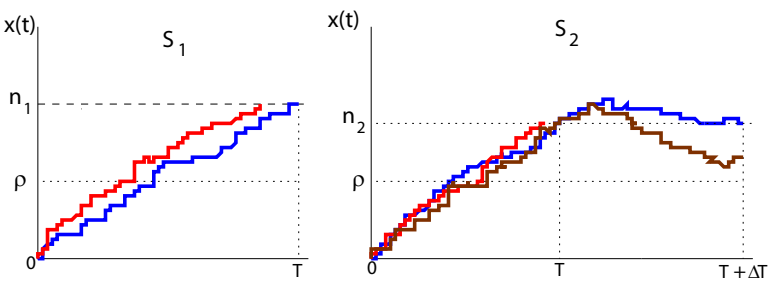

Figure 4: Sample paths for the process $x(t)$ in the two strategies $S_{1}$ and $S_{2} . \rho$ is the equilibrium value of the number of phosphorylated TCRs, $n_{i}$ are the thresholds in each strategy. In $S_{1}$ we are interested in sample paths that first reach the threshold $n_{l}$ before time $\leq T$ are indicated. In $S_{2}$ paths that first reach the threshold $n_{2}$ in time $\leq T$ and then retaining the threshold for a period $\leq \Delta T$ are indicated.

Triggering TCRs form a queue: The signalling events generated from the fully phosphorylated TCRs can be formulated as a queue. The fully phosphorylated TCRs start triggering with rate $\lambda$ and they down-regulate (removed) with rate $\mu x$.

The two strategies in terms of associated probabilities were rewritten:

- $\quad\left[\left(S_{1}\right)\right] \mathbb{P}\left[x\left(t^{\prime}\right) \geq \alpha_{1} R \mid x(0)=0, x(t)<\alpha_{1}\right.$ for $t<t^{\prime}$ $t<t^{\prime}$ and $\left.0<t^{\prime}<T\right]$ for some (given) $T$; i.e. the probability of first crossing the level $\alpha_{1} R$ computed.

- $\quad\left[\left(S_{2}\right)\right] \mathbb{P}\left[x(t) \geq \alpha_{2} \mid x(0)=0, x\left(t^{\prime}\right)=\alpha_{2}, t^{\prime} \leq T\right.$ is first crossing time and $T<t \leq \tau$ ] for some (given) $\tau$.

In this formalism, the TCR ${ }^{*}$ s start triggering as a Poisson process with rate $\lambda$ and the duration of an individual TCR signal (or service time) is exponential with parameter proportional to $x(t)$ - the number of TCRs involve in signalling at time $t$. The process $x(t)$ is a jump Markov process, which is the difference between two independent Poisson processes with rates $\lambda$ and $\mu x(t)$ with jumps of size +1 in direction +1 , and with jumps of size +1 in direction -1 respectively (see Figure 4). Let us write $n_{1}=\alpha_{1} R$ and $n_{2}=\alpha_{2} R$

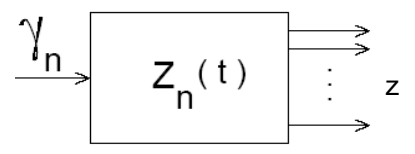

Figure 5 : The Scaled Process $z_{R}(t)$.

\section{RESULTS}

\section{Most probable path and large deviations}

T-cell activation under weak agonists is of low probability or is a rare event. The sample paths shown in Figure 4 are rare paths or those that have deviated from their equilibrium path. However, rare events occur in the most probable way. Thus, the most probable path that $x(t)$ will use to escape from its equilibrium path to cross the level $\alpha_{i}(i-1,2)$ was sought.

Instead of looking at the probability $\mathbb{P}\left[x(t) \geq n \alpha_{i}\right]$, $\mathbb{P}\left[z_{R}(t) \geq \alpha_{i}\right]$ was estimated where $z_{R}(t)=\frac{1}{R} x(t)$ is known as the scaled process. For the scaled process the incoming rate is $\gamma:=\lambda / R$, the outgoing rate is just $z(t)$.

This is exactly a standard Erlang queue (Shwartz \& Weiss, 1994). So, the scaled process $z_{R}(t)$ has jump rates $+1 / R$ with rate $\gamma R$ and jump rates $-1 / R$ with rate $z$.

The large deviation theory indicates that the probabilities associated with rare events decay exponentially with rate given by the so called rate function $I$ depending on parameters such as $T, \alpha_{i}, \lambda$, and $\tau$. It estimates the rare probabilities as

$\mathbb{P}\left[z_{R}(t) \geq \alpha_{i}\right] \approx \mathrm{e}^{-R I}$

Thus, an estimate of the above probability can be obtained by computing the rate function.

Applying Friedlin-Wentzell theory (Shwartz \& Weiss, 1994) to find the most probable path using large deviations, the most probable path is found as the one, which minimizes the associated cost in reaching the threshold $\alpha_{i}$ where the cost is defined by $\int l\left(t, r(t), r^{\prime}(t)\right) d t$ for any admissible path $r(t)$. Then the minimum cost is obtained via solving the variational problem given by

$I\left(\alpha_{i}\right)=\min _{r(t)} \int_{0}^{t_{i}} l\left(r(t), r^{\prime}(t)\right) d t$

where

$l\left(r, r^{\prime}\right)=r^{\prime} \log \frac{r^{\prime}+\sqrt{r^{\prime}+4 \gamma r}}{2 \gamma}+\gamma+r-\sqrt{r^{\prime}+4 \gamma r}$.

Here $t_{1}=T$ and $t_{2}=\tau$.

\section{Most probable path for activation in strategy $S_{1}$}

Proposition 1. The most probable path for $x(t)$ to first cross the threshold $n_{1}$ in time $T$ is given by 
$r(t)=k_{b}\left(\mathrm{e}^{t}-1\right)+\gamma\left(1-\mathrm{e}^{-t}\right), \quad k_{b}=\frac{\alpha_{1}-\gamma\left(1-\mathrm{e}^{-T}\right)}{\mathrm{e}^{T}-1}$

Proof. We take $\alpha_{1}>\gamma$, the initial/end conditions $r(0)=0$ and $r(T)=\alpha_{1}$ with $r^{\prime}(0)>0$ and $r^{\prime}(t)>0$ for all $t$.

First consider $k>0$ : Only $(+)$ ve solutions are admissible and using the initial/end conditions it is found

$r(t)=k\left(\mathrm{e}^{t}-1\right)+\gamma\left(1-\mathrm{e}^{-t}\right), \quad k=\frac{\alpha_{1}-\gamma\left(1-\mathrm{e}^{-T}\right)}{\mathrm{e}^{T}-1}>0$

$k<0$ yields only (-)ve solutions and so

$r(t)=k\left(1-\mathrm{e}^{t}\right)+\gamma\left(\mathrm{e}^{t}-1\right), \quad k=\frac{\alpha_{1}-\gamma\left(1-\mathrm{e}^{T}\right)}{1-\mathrm{e}^{-T}}<0$

For weak variations with respect to $T$ we see that $\quad \partial I / \partial T=l-r^{\prime} l_{r^{\prime}}=-k<0 \quad$ if $k>0$. Thus for $k>0$, the cost decreases as $T$ increases, and the opposite happens with $k<0$ solutions. Therefore, we conclude that the minimizing solution on $(0, T)$ is given by equation 3 .

\section{Most probable path in $S_{2}$}

The most probable path for the system after exiting the level $\alpha_{2}$ at time $T$ and reaching a level $\beta \geq \alpha$ at time $\tau$ with $r(t) \geq \alpha$ for all $t \in[T, \tau]$ with $r(T)=\alpha$ and $r(\tau)=\beta$ was determined as follows.

As the systems behaviour after reaching the level $\alpha_{2}$ is independent of its behaviour on $[0, T]$ the problem was split into the two domains: the solution on $[0, T]$ and the solution on $[T, \tau]$. The solution on the first half is identical to that in $S_{1}$ with the threshold $\alpha_{2}$. So the optimal path on $[T, \tau]$ needed to be determined.

Specifically, the variational problem needed to be solved

$\min _{r(t)} \int_{T}^{\tau} l\left(t, r(t), r^{\prime}(t)\right) d t$

with $l\left(t, r, r^{\prime}\right)$ being the same rate function found in equation 2 .

The local rate function $l(x, y)$ is defined in the usual manner

$$
l(x, y)=\sup _{\theta}\left\{\langle\theta, y\rangle-\log \mathbb{E}\left[\mathrm{e}^{\langle\theta, X(t)\rangle}\right]\right\}
$$

Note that since $x(t)=\sum x_{i}(t)$ where $\left\{x_{i}(t)\right\}$ is the Poisson jump process associated with the triggering events, it follows that

$$
l(x, y)=\sup _{\theta}\left\{\theta y-\gamma\left(\mathrm{e}^{\theta}-1\right)-x\left(\mathrm{e}^{-\theta}-1\right)\right\}
$$

where $\gamma=\lambda /(\mu R)$. The maximizer for $l(x, y)$ is

$\theta^{*}=\log \frac{1}{2 \gamma}\left(y \pm \sqrt{y^{2}+4 \gamma x}\right)$

So we find,

$$
l(x, y)=y \log \frac{1}{2 \gamma}\left[y+\sqrt{y^{2}+4 \gamma x}\right]+\gamma+x-\sqrt{y^{2}+4 \gamma x}
$$

To solve the variational problem the Euler-Lagrange equation of $l\left[t, r(t), r^{\prime}(t)\right]$ was considered. Since $l$ is not explicitly depending on $t$ there exists a first integral. Thus,

$$
l-r^{\prime} l_{r^{\prime}}=\gamma+r-\sqrt{r^{\prime}+4 \gamma r}=-k(\text { constant })
$$
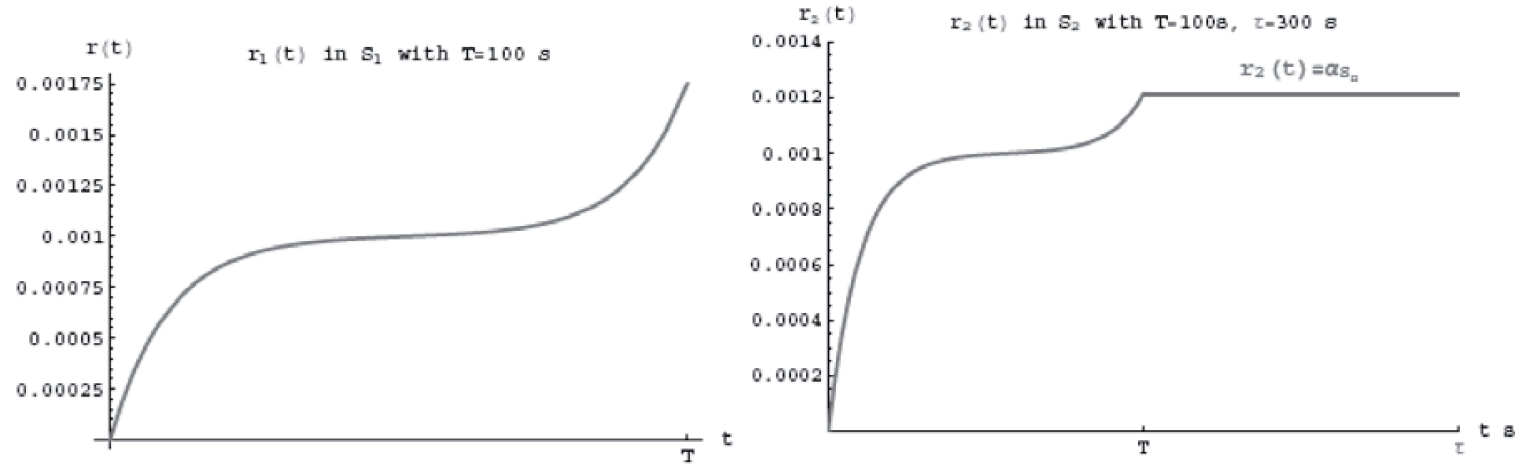

Figure 6 : Optimal paths $r_{1}(t)$ (left) and $r_{2}(t)$ (right) in strategies $S_{1}$ and $S_{2}$ respectively 
This yields $r^{\prime}= \pm\left[(r+k-\gamma)^{2}+4 \gamma k\right]^{1 / 2}$ which has the basic $C^{\infty}$ solution given by

$$
r(t)= \begin{cases}\gamma-k \pm \sqrt{|4 \gamma k|} \cosh (t+B) & k<0 \\ \gamma-k \pm \sqrt{4 \gamma k} \sinh (t+B) & k>0\end{cases}
$$

where $B$ is arbitrary.

If $k=0$ we have

$r(t) \equiv r_{e_{ \pm}}(t)=\gamma+\mathrm{e}^{B \pm t}$

which is called the equilibrium solution.

Proposition 2. Let $r(t) \geq \alpha_{2}$ for $t \in[T, \tau]$ such that $r(T)=\alpha ;$ and $r(\tau)=\beta$. Then the optimal path for the variational problem (equation 4) is given by $r_{0}(t)=\alpha_{2}$

Proof. Notice that the admissible paths should satisfy the following conditions:

- $r^{\prime}(T) \geq 0$,

- $r(T)=\alpha_{2}, r(\tau)=\beta \geq \alpha_{2}$,

- $r(t) \geq \alpha_{2}$ for $T \leq t \leq \tau$

and these admissible paths are

(i) $r_{0}(t)=\alpha_{2}$

(ii) $r_{1}(t)=\gamma-k-\sqrt{4|k| \gamma} \cosh (t+B)$ with $B<0$

(iii) $r_{2}(t)=\gamma-k+\sqrt{4|k| \gamma} \cosh (t+B)$ with $B>0$

(iv) $r_{3}(t)=\gamma-k+\sqrt{4|k| \gamma} \sinh (t+B)$ with $B \gtrless 0$

(v) $k \rightarrow 0$ solution: $r_{e_{+}}(t)=\gamma+(\alpha-\gamma) \mathrm{e}^{t+B}$

Case I: $r_{0}(t)$ is a local minimum for all possible weak variations: Consider a weak variation of $r_{0}(t)$ given by $r(t)=r_{0}(t)+\delta h(t)$ such that $\delta h(T)=\delta h(\tau)=0$ and $\delta h(t)>0$ for $T \leq t \leq \tau$.

$\delta I=\left(1-\sqrt{\alpha_{2} \gamma}\right) \int_{T}^{\tau} \delta h d t>0 \quad$ since $\quad \alpha_{2}>\gamma$. This shows that $r_{0}(t)$ is a local minimum under the constraints $\delta h(T)=\delta h(\tau)=0$.

Next consider the case $\delta h(\tau)=: \delta \beta>0$ :

$\delta I=\log \sqrt{\gamma / \alpha_{2}}[\delta h]_{T}^{\tau}+\left(1-\sqrt{\frac{\gamma}{\alpha_{2}}}\right) \int_{T}^{\tau} d t$ is obtained

Since both terms are positive, it follows that $\delta I>0$.
Hence, $r_{0}(t)$ is a local minimum under all allowable variations.

Case II: The author proves that

\section{(a) $I_{2}\left[r_{1}\right]>I_{2}\left[r_{0}\right]$ :}

$r_{1}(t)=\gamma-k-\sqrt{4|k| \gamma} \cosh (t+B)$ with $B<0$ is obtained. As $\partial I_{2} / \partial \beta<0$ for all negative $\cosh$ (as well as negative sinh solutions), it is anticipated that there exists some $\beta_{\max }>\beta$ at which $I_{2}$ attains its minimum if it exists. By considering $r(\tau)=\beta$ it can be written

$B=-(\tau-T) \pm \cosh ^{-1}\left(\frac{1}{2} \sqrt{\frac{x}{\gamma}}-\frac{\beta-\gamma}{\sqrt{4 x \gamma}}\right)$

where $\quad x=-k>0$. By observing that as $\beta \rightarrow \infty, B \rightarrow-\infty$ and $x=-k \rightarrow \infty$, for $B$ large and negative

$r(t) \approx \gamma-k-\sqrt{\gamma x} \mathrm{e}^{-t+T} \mathrm{e}^{B}$ is obtained.

Using $r(T)=\alpha_{2}$ and $r(\tau)=\beta$ it is found

$r \approx \gamma-k-\frac{\beta-\alpha}{1-\mathrm{e}^{-\tau+T}} \mathrm{e}^{-t+T}$

and so,

$$
\begin{aligned}
I_{2}\left[r_{1}\right] & =\int_{T}^{\tau} r_{1}^{\prime}(t) l_{r_{1}^{\prime}}(t) d t-k(\tau-T) \\
& =\int_{T}^{\tau} \frac{\beta-\alpha}{1-\mathrm{e}^{-\tau+T}} \mathrm{e}^{-t+T} \log \left(1-\sqrt{\frac{|k|}{\gamma}} \mathrm{e}^{t+B}\right) d t-k(\tau-T) \\
& \approx|k|\left(\mathrm{e}^{T}-1\right)(\tau-T)
\end{aligned}
$$

Since $I_{0}(\tau)=(\tau-T)(\sqrt{\alpha}-\sqrt{\gamma})^{2}$

$I_{0} / I_{2} \approx(\sqrt{\alpha}-\sqrt{\gamma})^{2} / x\left(\mathrm{e}^{T}-1\right)<1$ as $x \rightarrow \infty$ is obtained for $\beta$ large.

(b) $I_{e}:=I_{2}\left[r_{e_{+}}\right]>I_{2}\left[r_{0}\right]=: I_{0}$ :

Without loss of generality let us take $T=0$.

$$
\begin{aligned}
I_{e}= & \int_{0}^{\tau} r_{e^{+}}^{\prime} l\left(t, r_{e^{+}}, r_{e^{+}}^{\prime}\right) d t \\
= & \int_{0}^{\tau}(\alpha-\gamma) \mathrm{e}^{t} \log \left(1+\frac{(\alpha-\gamma)}{\gamma} \mathrm{e}^{t}\right) \\
= & \left(1-\mathrm{e}^{\tau}\right)(\alpha-\gamma)(1+\log \gamma)-\alpha \log \alpha+ \\
& \quad\left(\mathrm{e}^{\tau}(\alpha-1)+\gamma\right) \log \left(\mathrm{e}^{\tau}(\alpha-\gamma)+\gamma\right) .
\end{aligned}
$$


By observing that $\left.I_{e}\right|_{\tau=0}=\left.I_{0}\right|_{\tau=0}=0$ and that

$\frac{d I_{e}}{d \tau}=\mathrm{e}^{\tau}(\alpha-\gamma) \log \left[1+\mathrm{e}^{\tau}\left(\frac{\alpha}{\gamma}-1\right)\right]>\frac{d I_{0}}{d \tau}>0$

for all $\tau \geq 0$ it can be concluded that $I_{e}>I_{0}$ for all $T \leq t \leq \tau$.

Case III: It is easy to see that $\partial I / \partial \beta=l_{r^{\prime}}>0$ for the paths with (-)ve signs i.e. (iii) and (iv) above. This means that the minimum should attain as $\beta \rightarrow 0$ with respect to those solutions. $\beta \rightarrow 0$ is possible with either $r_{1}(t)$ with $B=-(\tau-T) / 2$ or with $r_{0}(t)$. But by Case II above, we know that $r_{0}(t)$ is optimal against $r_{1}(t)$.

The above three cases show that the optimal solution is indeed $r_{0}(t)$.

The optimal paths computed according to Propositions 1 and 2 for $T=100 \mathrm{~s}$ and $\tau=300 \mathrm{~s}$ are shown in Figure 6.

\section{DISCUSSION AND CONCLUSION}

When does the activation of a T-cell become hard (or rare)? If the T-cells fail to respond to invading pathogens within reasonable time (for which we have enough evidence: i.e. failure of the immune system to recognize the HIV), then the activation of a T-cell becomes hard (or rare). Indeed, the HIV specifically targets the CD4 molecule of human T-cells. The role of T-cell activation when the CD4 molecule is inhibited has been reported by Irvine and Davis (Irvine \& Davis, 2002) where this probability lies in the range $0.001-0.002$.

Large deviation theory provides us with a powerful tool to estimate the probabilities of so called rare events. In the context of the T-cell activation this technique was used to compute the most probable way (or the path) the T-cell could achieve a given cellular level threshold leading to its activation as defined in the text in different contexts, in two different strategies.

Besides, in situations where the activation happens with high probability, one could formulate the problem considering the probabilities of the events in the complementary space for which the probabilities are relatively small (i.e. if the event $E$ is not rare the large deviation theory is used to compute the probability $1-\mathbb{P}\left(E^{c}\right.$ where the superscript $c$ stands for the complement).

The results found in the present modelling exercise are summarized in Figure 7 where the response of the T-cells in the two strategies are shown via the associated activation probability $P(\lambda)$ as a function of the triggering rate $\lambda$ of the T-cell, associated in the two strategies computed numerically via equation 1 . It is clear from these plots that in the second strategy $-S_{2}$, the T-cell response to agonists have become narrower (which has taken 5 hours from the initial cell-cell contact), whence in the first strategy - $S_{1}$, T-cells respond to a broad spectrum of agonists for which it has taken 1 hour from the initial cell-cell contact. However, when the CD4 functions normally, a T-cell shall be able to react within

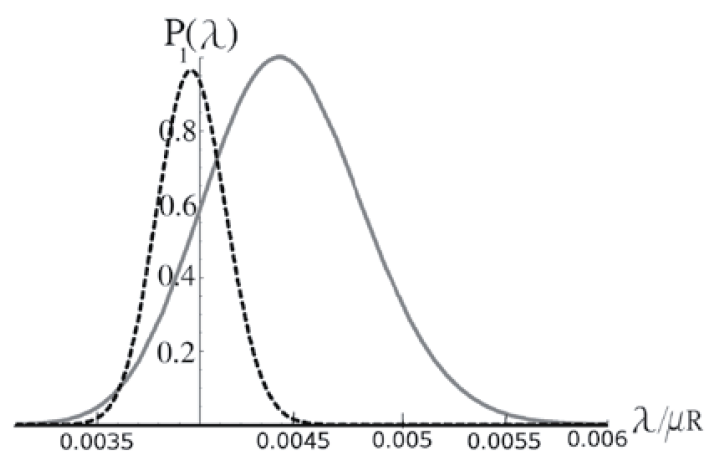

Figure 7 : Probability of activation as a function of $\lambda$ which is the rate at which the TCRs 'join' in a pool of signaling TCRs. The solid line corresponds to the strategy $S_{1}$ described in the text and the broken line to strategy $S_{2}$. Notice the peaked behaviour in the second case which shows that the T-cells have a highly specific response to agonists which takes place after about 6 hours from initial contact with the APC. In contrast, the T cell responds to a broad range of agonists within the first 1 hour. (Parameters are taken as follows: corresponding to each strategy for parameters $n=30000, \gamma=0.0158198, r=.001 s^{-1}$, Number of agonists $M=10, T=1$ hour, $\tau=1+\Delta T$ (hours) with $\Delta T=5$ hours. Parameters taken from Davis, 2009. 
30 seconds (Irvine \& Davis, 2002), which accounts for early $\mathrm{Ca}^{+}$signals observed. The present study has been able to provide an answer to the questions as to how long an average T-cell would require to get into early stages of its activation (i.e. initiating $\mathrm{Ca}^{+}$signalling), for instance in patients infected by HIV as it is the CD4 receptor that becomes the primary target of the HIV (Traunecker et al., 1988; McMichael et al., 2010).

The thresholds in strategies $S_{1}$ and $S_{2}$ i.e. $\alpha_{1}=$ 0.01149 and $\alpha_{2}=0.01090$ respectively are computed in each case by solving $\log \left[\mathbb{P}\left(\right.\right.$ activation in $\left.\left.S_{i}\right)\right] \approx-n I_{i}$ $i=1,2 . I_{1}$ and $I_{2}$ are the rate functions obtained in previous sections.

\section{Acknowledgment}

The author is thankful to the National Research Council of Sri Lanka for providing financial support for this project under the Grant No. NRC/05/64.

\section{References}

1. Bossi G., Trambas C., Booth S., Clark R., Stinchcombe J. \& Griffiths G.M.(2002). The secretory synapse: the secrets of a serial killer. Immunological Reviews 189:152-160.

2. Chan C., George A.J.T. \& Stark J.(2001). Cooperative enhancement of specificity in a lattice of $\mathrm{T}$ cell receptors. Proceedings of the National Academy of Sciences of the United States of America 98(10): 5758-5763.

3. Chao D. L., Davenport M.P., Forrest S. \& Perelsond A. S. (2003). A stochastic model of cytotoxic t cell responses. Journal of Theoretical Biology 228:227-240.

4. Irvine D. J., Purbhoo M. A., Krogsgaard M. \& Davis M. M. (2002). Direct observation of ligands recognition by $\mathrm{t}$ cells. Nature 419:845-848.

5. Davis D. M.(2009). Mechanisms and functions for the duration of intercellular contacts made by lymphocytes. Nature Reviews Immunology 9:543-555.

6. Dustin M.L., Bivona T.G. \& Philips M.R.(2004). Membranes as messengers in $\mathrm{T}$ cell adhesion signaling. Nature Immunology 5:363-372.

7. Faroudi M., Zaru R., Paulet P., Müller S. \& Valitutti S. (2003). Cutting edge: $T$ lymphocyte activation by repeated immunological synapse formation and intermittent signaling. The Journal of Immunology 171:1128-1132.

8. Freidlin M.I. \& Wentzel A.D. (1984). Random Perturbations of Dynamical Systems. Springer-Verlag, New York, USA.

9. Gett A. V., Sallusto F., Lanzavecchia A. \& Geginat J.(2003). T-cell fitness determined by signal strength. Nature Immunology 4:355-360.
10. Gunzer M., Schafer A., Borgmann S., Gragge S., Zanker K.S., Brocker E.B., Kampgen E. \& Friedl P.(200). Antigen presentation in extracellular matrix: interactions of T-cells with dendritic cells are dynamic, short lived, and sequential. Immunity 13: 323.

11. Hemmer B., Stefanova I., Vergelli M., Germain R.N. \& Martin R. (1998). Relationships among TCR ligand potency, thresholds for effector function elicitation, and the quality of early signaling events in human t cells. The Journal of Immunology 160:5807-5814.

12. Hopfield J.J.(1974). Kinetic proofreading: a new mechanism for reducing errors in biosynthetic processes requiring high specificity. Proceedings of the National Academy of Sciences of the United States of America 71:4135-4139.

13. Huppa J.B., Gleimer M., Sumen C. \& Davis M.M.(2003). Continuous $\mathrm{T}$ cell receptor signaling required for synapse maintenance and full effector function. Nature Immunology 4: 749-755.

14. Itoh Y., Hemmer B., Martin R. \& Germain R.N.(1999). Serial TCR engagement and down-modulation by peptide:MHC molcule ligands: relationship to the quality of individual TCR signalling events. The Journal of Immunology 162:2073-2080.

15. Jordan M. S., Smith-Garvin J. E. \& Koretzky G. A. (2009). T-cell activation. Annual Review of Immunology 27:591-619.

16. Kaech S.M. \& Ahmed R. (2001) . Memory CD8 ${ }^{+}$T-cell differentiation: initial antigen encounter triggers a developmental program in naive cells. Nature Immunology 2:415-422.

17. Kersh G.J., Miley M.J., Nelson C.A., Grakoui A., Horvath S., Donermeyer D.L., Kappler J., Allen P.M. \& Fremont D.H.(1998). Structural and functional consequences of altering a peptide MHC anchor residue. The Journal of Immunology 166:3345-3354.

18. Lanzavecchia A. (1997). Understanding the mechanisms of sustained signaling and t cell activation. The Journal of Experimental Medicine 185(10):1717 -1719.

19. McKeithan T.W. (1995). Kinetic proofreading in T-cell receptor signal transduction. Proceedings of the National Academy of Sciences of the United States of America 92:5042-5046.

20. McMichael A. J., Borrow P., Tomaras G. D. \& Goonetilleke N. (2010). The immune response during acute HIV-1 infection: clues for vaccine development. Nature Reviews Immunology 10:11-23.

21. Noest A. (2000). Designing lymphocyte functional structure for optimal signal detection: Viola, T-cells. Journal of Theoretical Biology 207(2): 195-216.

22. Parham P.(2000). The Immune System. Garland Publishing, New York, USA.

23. Roitt I. (1998). Immunology: Mosby-Year Book, $5^{\text {th }}$ edition. The Open University Press, Milton Keynes, UK.

24. Schrum A.G. \& Turka L.A. (2002). The proliferative capacity of individual naive $\mathrm{CD} 4^{+} \mathrm{t}$ cells is amplified by prolonged $\mathrm{T}$ cell antigen receptor triggering. The Journal of Experimental Medicine 196:793-803.

25. Shwartz A. \& Weiss A. (1994). Large Deviations for 
Performance Analysis. Chapman \& Hall Publishers, London, UK.

26. Traunecker A., Lüke W. \& Karjalainen K. (1998). Soluble CD4 molecules neutralize human immunodeficiency virus type 1. Nature 331:84-86.

27. Valitutti S. \& Lanzavecchia A.(1997). Serial triggering of TCRs: a basis for the sensitivity and specificity of antigen recognition. Immunology Today 18:299-304.

28. Valitutti S., Dessing M., Aktories K., Gallati H. \& Lanzavecchi A. (1995). Sustained signalling leading to T-cell activation results from prolonged T-cell receptor occupancy. role of the actin cytoskeleton. Immunology Today 181:577-584.

29. Valitutti S., Muller S., Cella M., Padovan E. \& Lanzavecchi A. (1995). Serial triggering of many T-cell receptors by a few peptide-MHC complexes. Nature 375:148-151.

30. Valitutti S., Muller S., Dessing M. \& Lanzavecchi A.(1996). Signal extinction and T-cell repolarization in T helper cell-antigen-presenting cell conjugates. European Journal of Immunology 26:2012-2016.

31. Valitutti S., Muller S., Dessing M. \& Lanzavecchia A. (1996) . Different responses are elicited in cytotoxic $\mathrm{T}$ lymphocytes by different levels of $\mathrm{t}$ cell receptor occupancy. The Journal of Experimental Medicine 183:1917-1921.
32. van Bergen J., Kooy Y. \& Koning F.(2001). CD4independent $\mathrm{T}$ cells impair TCR triggering of CD4dependent $\mathrm{T}$ cells: a putative mechanism for $\mathrm{T}$ cell affinity maturation. European Journal of Immunology 31:646-652.

33. van den Berg H.A., Rand D.A. \& Burroughs N.J.(2001). A reliable and safe T-cell repertoire based on lowaffinity T-cell receptors. Journal of Theortical Biology 209 (4):465-486.

34. van Stipdonk M. J. B., Hardenberg G., Bijker M. S., Lemmens E. E., Droin N. M., Green D. R. \& Schoenberger S.P.(2003). Dynamic programming of $\mathrm{CD}^{+}$lymphocyte response. Nature Immunology 4:361-365.

35. van Stipdonk M. J. B., Lemmens E. E. \& Schoenberger S.P.(2001). Naive CTLs require a single brief period of antigenic stimulation for clonal expansion and differentiation. Nature Immunology 2:423-429.

36. Wedagedera J. R. \& Burroughs N.J.(2009). Two-level strategy for T-cell activation: a large deviation analysis for CD4 blocked conditions. Journal of Biological Systems (Accepted for publication, January 2011).

37. Palmer E. \& Werlen G.(2002). The TCR signalosome: a dynamic structure with expanding complexity. Current Opinion in Immunology 14(1):299-305. 